\title{
Use of Space Thermal Factors by Spacecraft
}

\author{
N. Semena \\ Space Research Institute RAS, \\ Russia
}

\section{Introduction}

All the equipment used by the man can work in the limited temperature range. The necessary ambient temperature and the intensive convective mechanism help to solve a thermostabilization problem on the Earth. But in the space the decision of this problem is much more difficult by reason of the extreme thermal conditions and vacuum. Now thermostabilization of space devices is provided with special thermoregulation systems, which failure leads to emergency end of mission. These systems depend from spacecraft (Sc) electrical system which supplies energy heaters and from Sc orientation system, which doesn't allow to heat up a radiator solar or planetary irradiance.

In article it will be shown that using of very simple technical decisions allows to make Sc thermoregulation systems independent of other Sc systems and from variation of space thermal factors. In addition it is shown how Sc thermal systems can be used for determine of its orientation.

\section{Analysis of shortcomings of the conventional system for ensuring the thermal regime}

To solve the problem of thermal stabilization of space equipment sufficiently efficient systems of thermal regulation were developed whose basic elements are the radiatoremitter, which is a surface emitting the excessive heat flux to space, and the electric heater - the element heating the equipment if necessary.

The process for maintaining the temperature of an equipment used in space generally consists of the maintenance of a necessary temperature level of the heat balance between the heat flux irradiated from the radiator surface and the integral heat capacity of the device including heat release of the equipment, heat release of the heater and the heat flux absorbed by the external surface of the radiator-emitter. The scheme of the simplest system of thermal regulation is presented in Fig. 1.

To investigate the influence of external and internal thermal factors on the temperature regime of such a system one can use an assessment thermal model which does not account for secondary factors: the non-isothermicity of thermal nodes, heat flux across the external thermal insulation, the difference from zero of the effective temperature of space, and a possible shielding of the radiator-emitter by the structure external elements. The above factors do not affect the qualitative result of modelling but complicate the solution. Thus, the 


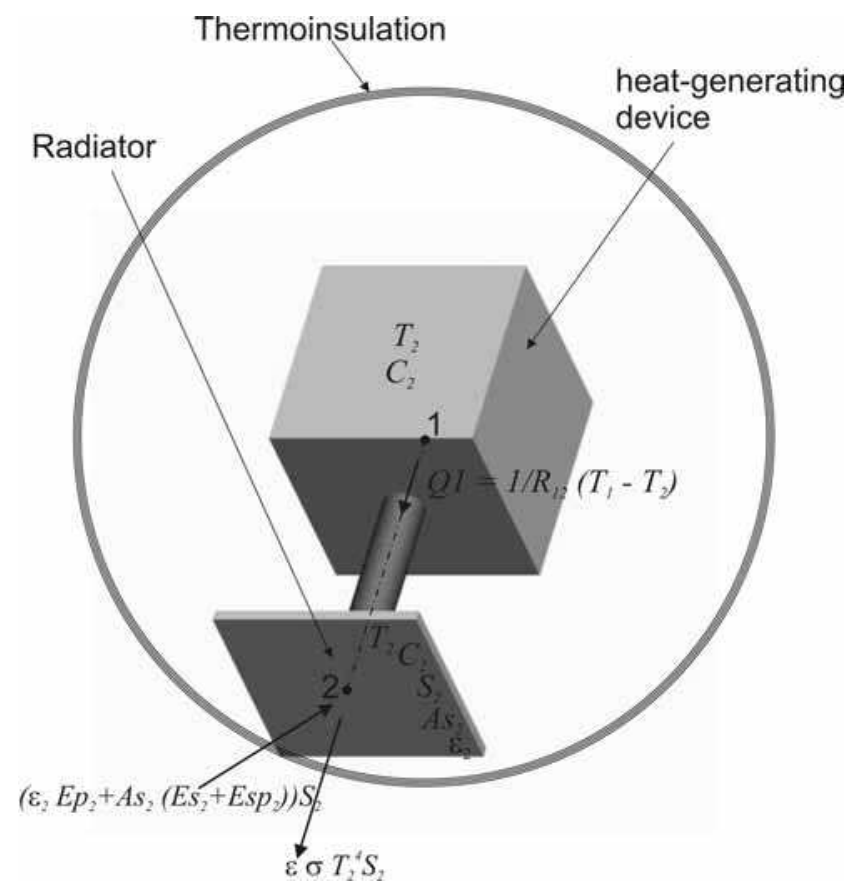

Fig. 1. Scheme of a conventional system for thermal regulation of the devices for space application.

assessment thermal model of the presented system includes two thermal nodes (node No. 1 is the heat releasing equipment, including the heater, nodes No. 2 is the radiator-emitter) and is governed by the system of two equations:

$$
\begin{aligned}
& C_{1} \frac{d T_{1}}{d \tau}=\left(Q_{1}+Q_{H}\right)-\frac{1}{R_{12}}\left(T_{2}-T_{1}\right) \\
& C_{2} \frac{d T_{2}}{d \tau}=\left(\varepsilon_{2} E p_{2}+A s_{2}\left(E s_{2}+E s p_{2}\right)\right) S_{2}+\frac{1}{R_{21}}\left(T_{1}-T_{2}\right)-\varepsilon_{2} \sigma T_{2}^{4} S_{2},
\end{aligned}
$$

where $C_{1}, C_{2}, T_{1}$, and $T_{2}$ are the heat capacities and temperatures of the device and the radiator, $\tau$ is the time, $Q_{1}$ and $Q_{H}$ are the heat releases of the device and the heater, $S_{2}, \varepsilon_{2}$ and $A s_{2}$ are the area, emissivity factor, the coefficient of absorption of solar radiation and the external surface of the radiator-emitter, $E p_{2}$ and $E s_{2}+E s p_{2}$ are the infrared and solar radiant fluxes incident onto the external surface of the radiator-emitter, $R_{12}$ and $R_{21}$ are the thermal resistance of the heat-conducting duct from the equipment to radiator and from the radiator to the equipment (usually $R_{12}=R_{21}$ ), $\sigma$ is the Stefan - Boltzmann constant.

An analysis of the presented thermal model shows the shortcomings of the conventional system for ensuring the thermal regime, which is employed in present-day devices of space application.

1. Such a system is very sensitive to external heat fluxes falling onto the radiator- emitter surface. The reason for this is that the only model element, at the expense of 
which the heat is removed is $\varepsilon 2 \sigma \mathrm{T} 24 \mathrm{~S} 2$, therefore, the system can function efficiently only at such a level of external heat fluxes (Ep, Es+Esp), which ensure, for a given temperature of the equipment (T1), the satisfaction of inequality $(\varepsilon 2 \mathrm{Ep} 2+$ As2(Es2+Esp2))S2 $\ll$ At the equality of these two elements, the radiator-emitter stops functioning, and at a sign change of the inequality to the opposite sign the radiator-emitter reverts into a heater and stabilizes the system at a higher temperature as compared to the one, which is required for the equipment operation. This indeed means that the spacecraft must not be oriented in such a way that a highly intense external flux from the sun and a planet falls during a long time onto the radiator-emitter. The orientation constraints in their turn lead to a restriction of the spacecraft functional capabilities.

2. The system is sensitive to the internal heat release of the equipment because a small oscillation of temperature around the mean value is ensured only under the condition $Q_{1}+$ $Q_{H} \approx$ const. This means that at a reduction of the useful power consumption of equipment the freed power must be directed to the heater feeding to maintain a constant level of the total heat release. This leads, in its turn, to the fact that the power supply system of spacecraft must always be tuned to a peak power consumption, which is very wasteful under the conditions of an electric power shortage on the spacecraft.

A seeming possibility of the first factor compensation at the expense of the second one leads to an even higher loading on the power supply system, which must compensate in this case both for a non-uniformity of the internal heat release of the equipment and non-uniformity of the external radiant flux absorbed by the radiator-emitter. Thus, if a spacecraft is composed of several independent devices each of which is equipped with an autonomous system for ensuring the thermal regime and the given devices are switched on at different times, then one can ensure, at first glance, the electric power saving by directing it only to those devices, which must be switched on. However, this is impossible when using the conventional systems for ensuring the thermal regime because the specified temperature of the equipment is ensured only at a constant maximum power supply to each device.

\section{Universal mechanism of self-regulation}

The self-regulation mechanism of a passive system for ensuring the thermal regime must ensure the temperature independence of thermally stabilized equipment of the external heat flux variability and of its internal heat release variability in the absence of active elements. At first sight, these are mutually exclusive conditions. If one considers, however, the spacecraft as an element included in the entire thermal balance of the Solar system, then one can conclude that the presence of a stable heating source, the sun, and a stable cooling source, the open space, enables the given problem solution.

If the radiator is partitioned into six parts oriented at the right angle with respect to one another (Fig. 2), then independently of the direction in which the sun or a planet lies the integral heat flux absorbed by six radiators will vary weakly:

$$
\sum_{i=2}^{i=7}\left(\varepsilon_{i} E p_{i}+A s_{i}\left(E s_{i}+E s p_{i}\right) S_{i} \approx \text { Const },\right.
$$

where $i$ is the radiator number. This leads in its turn to that the external radiant flux absorbed by radiators is taken into account at the choice of the radiator areas as a constant heat addition, which does not lead to oscillations of temperatures of the thermal model nodes. 


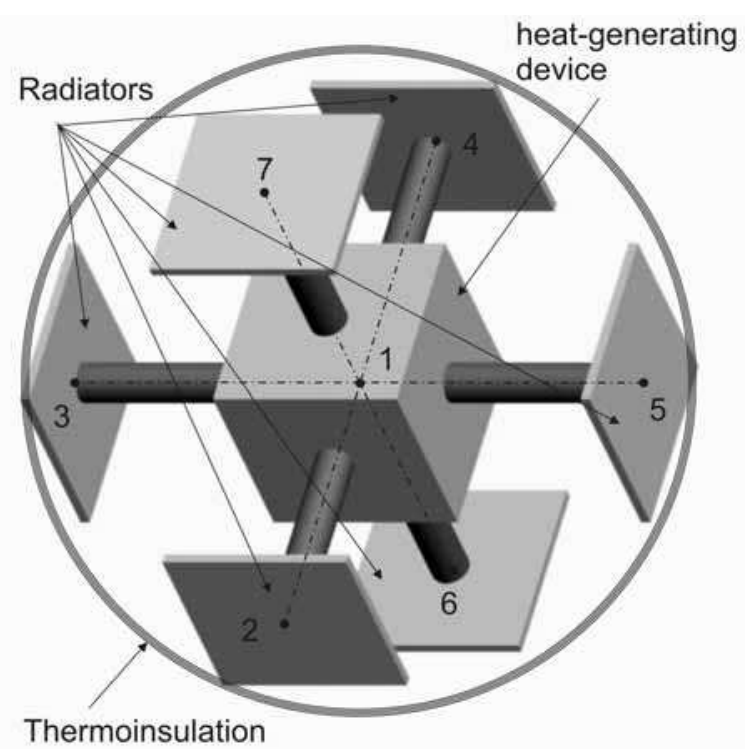

Fig. 2. Six-radiator system for ensuring equipment the thermal regime.

The given method is very efficient for compensation of the variability of radiant flux onto the spacecraft external surface under its arbitrary variable orientation.

This method also enables a partial compensation of the internal heat release variability. A constant external heat inflow into the system will enable the maintenance of the equipment temperature at a minimally allowed level even at its switch-off and a non- functioning heater, that is at $Q_{1}+Q_{H}=0$. The necessary heat balance may be ensured at the expense of choosing the optical characteristics $\left(A s_{i}, \varepsilon_{i}\right)$ of the external surface of radiators-emitters. The use of a many-radiator system enables one in some cases to refuse completely the use of heater.

As a rule, it is impossible to mount six radiators on a device because of a limited angular coefficient of the space survey and design constraints. Such a system may be used with a lesser number of radiators, but also with a lower efficiency.

\section{Special mechanisms of self-regulation}

The above presented mechanism of self-regulation is universal, it enables the maintenance of temperature of the heat-stabilized equipment within the given limits under the spacecraft orientation variation and under a drop of internal heat release, for example, at an accident switch-off of the equipment. There is, however, an equipment, which operates under specific thermal conditions, for example, under considerable single increases in heat release or at a very low level of temperatures. A simple solution using the separation and different orientation of radiators-emitters is insufficient for thermal stabilization of such an equipment. The advanced adjustable passive heat pipes, the gas- regulated heat pipes (GRHP) and thermal diodes (TD) [5], must be used within the system for ensuring the thermal regime of such an equipment. In the heat pipe, the heat transport occurs at the expense of the motion of evaporated heat-transfer agent from evaporation zone to 
condensation zone. The return of condensed heat-transfer agent to evaporation zone occurs at the expense of capillary forces. The heat pipe edge to which the heat flux is supplied is usually the evaporation zone, and the opposite edge is the condensation zone. The condensation zone may, however, shift in GRHP along the heat pipe length depending on the value of a heat flux fed to the evaporation zone. Since effective heat transport is performed in the heat pipe only between the zones of evaporation and condensation, the GRHP represents a heat pipe of variable length. Thus, a radiator-emitter with a variable effective emissive area depending on the supplied heat flux value may be constructed based on a heat pipe and a plate with limited thermal conductivity (Fig. 3). The thermal diode is a heat pipe with a unidirectional conductivity. The given element may be used for a lowtemperature system of ensuring the heat regime, if there is a need in minimizing the reverse heat inflow from the radiator-emitter.

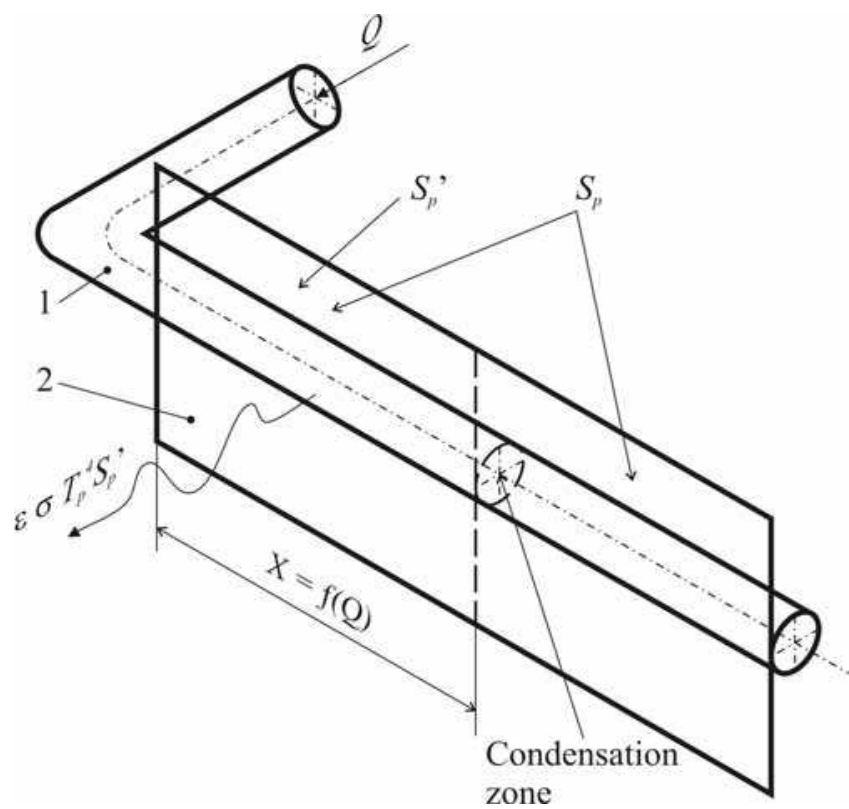

1 - GRHP, 2 - a plate with limited longitudinal thermal conductivity, $S_{P}{ }_{P}-$ the effective emissive area of the radiator (the active area), $S_{P}$ is the radiator maximum area, $T_{P}$ is the temperature of the radiator active zone, $X$ is the GRI-IP active part length

Fig. 3. Radiator with an adjustable effective emissive area.

\section{Efficiency of self-regulation mechanisms}

With regard for the partition of the radiator-emitter into separate differently directed elements, the introduction in the system for ensuring the thermal regime of adjustable radiators based on GRHP and the plates with limited thermal conductivity as well as the use of the TD, the assessment mathematical model of a passive system for ensuring the thermal regime with introduced self-regulation mechanisms will be as follows: 


$$
\begin{aligned}
& C_{1} \frac{d T_{1}}{d \tau}=Q_{1}-\sum_{i=2}^{n} \frac{1}{R_{1 i}\left(\Delta T_{i 1}\right)} \cdot\left(T_{i}-T_{1}\right) \\
& C_{2} \frac{d T_{2}}{d \tau}=\left(\varepsilon_{2} \cdot E p_{2}+A s_{2} \cdot\left(E s_{2}+E s p_{2}\right)\right) \cdot S_{2}\left(\Delta T_{21}\right)+\frac{1}{R_{21}\left(\Delta T_{12}\right)} \cdot\left(T_{1}-T_{2}\right)- \\
& -\varepsilon_{2} \cdot \sigma \cdot T_{2}^{4} \cdot S_{2}\left(\Delta T_{21}\right) \\
& \ldots \\
& C_{n} \frac{d T_{n}}{d \tau}=\left(\varepsilon_{n} \cdot E p_{n}+A s_{n} \cdot\left(E s_{n}+E s p_{n}\right)\right) \cdot S_{n}\left(\Delta T_{n 1}\right)+\frac{1}{R_{n 1}\left(\Delta T_{1 n}\right)} \cdot\left(T_{1}-T_{n}\right)- \\
& -\varepsilon_{n} \cdot \sigma \cdot T_{n}^{4} \cdot S_{n}\left(\Delta T_{n 1}\right),
\end{aligned}
$$

where $C_{1}, T_{1}$, and $Q_{1}$ are the heat capacity, the temperature, and heat release of the equipment, $\tau$ is the time, $i=2 \ldots \mathrm{n}$ is the number of radiators, $C_{i}$, and $T_{i}$ are the heat capacity and the temperature of the $i$-th radiator, $S_{i}\left(\Delta T_{i 1}\right)$ is the effective emissive area of the $i$-th radiator, which is a function of the heat flux to it or, what is the same, of the difference $T_{i}-T_{1}, \varepsilon_{i}$ and $A s_{i}$ are the emissivity factor, the coefficient of the solar radiation absorption by the external surface of the $i$-th radiator, $E p_{i}$ and $E s_{i}+E s p_{i}$ are the infrared and solar radiant fluxes falling onto the external surface of the $i$-th radiator, $R_{1 n}\left(\Delta T_{i 1}\right)$ and $R_{n 1}\left(\Delta T_{i 1}\right)$ are the thermal resistance of the heat-conducting duct from the equipment to the $i$-th radiator and from the radiator to the equipment, what in the case of using a thermal diode depends on the heat flux direction, or, what is the same, on the sign of the difference $T_{i}-T_{1}$.

All the self-regulation mechanisms are presented in the given model. It is enough to use in real systems one of the proposed techniques, which will be in terms of its characteristics the closest one to the requirements made by the thermally stabilized equipment.

The model was used for determining the efficiency of techniques proposed for selfregulation. To this end the real situations were modeled, which are critical for the conventional system of ensuring the thermal regime. An electronic block with the parameters typical of the present-day equipment was the thermal regulation object: its mass was $10 \mathrm{~kg}(C 1 \approx 900 \mathrm{~J} / \mathrm{K})$ and heat release $Q=10 \mathrm{~W}$. The conventional system of ensuring the thermal regime for such a block must have a radiator with area $S_{2}=0.03 \mathrm{~m}$ with optical characteristics $A s_{2}=0.2, \varepsilon_{2}=0.9$, provided that the solar radiation does not fall on the radiator. While using the universal self-regulation mechanism it is necessary to employ six radiators, each of which must have the following characteristics: $S_{i}=0.015, A s_{i}=0.9, \varepsilon_{i}=0.9$, $\mathrm{i}=2$....7.

Figure 4 shows the temperature variation of the thermal regulation object mounted on an orbital spacecraft (the time of a single revolution is $90 \mathrm{~mm}$ ) at an orbit turn of $90^{\circ}$ with respect to the direction to the sun (it occurs at the expense of the orbit precession). The application of six radiators in this situation is seen in Fig. 4 to enable the preservation of the thermal regulation object temperature within the range $21.7 \pm 0.1^{\circ} \mathrm{C}$, whereas in the case of using a single radiator the temperature increases from 21 to $38^{\circ} \mathrm{C}$. 


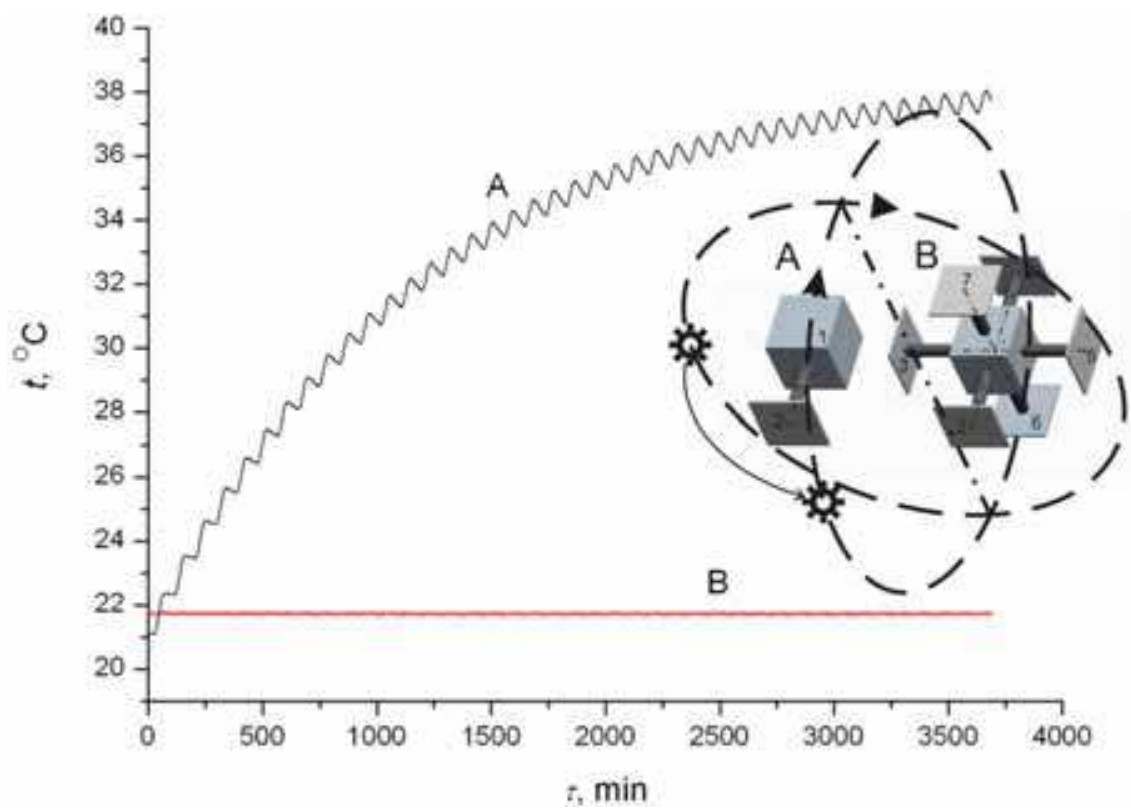

The systems supplied with radiators: one radiator (A), six radiators (B).

Fig. 4. Temperature variation of the thermal regulation object at an orbit rotation of the spacecraft.

Figure 5 shows the temperature drop of the same object at a switch-off of the electric power during $900 \mathrm{~mm}$ (a possible situation at the electric power shortage).

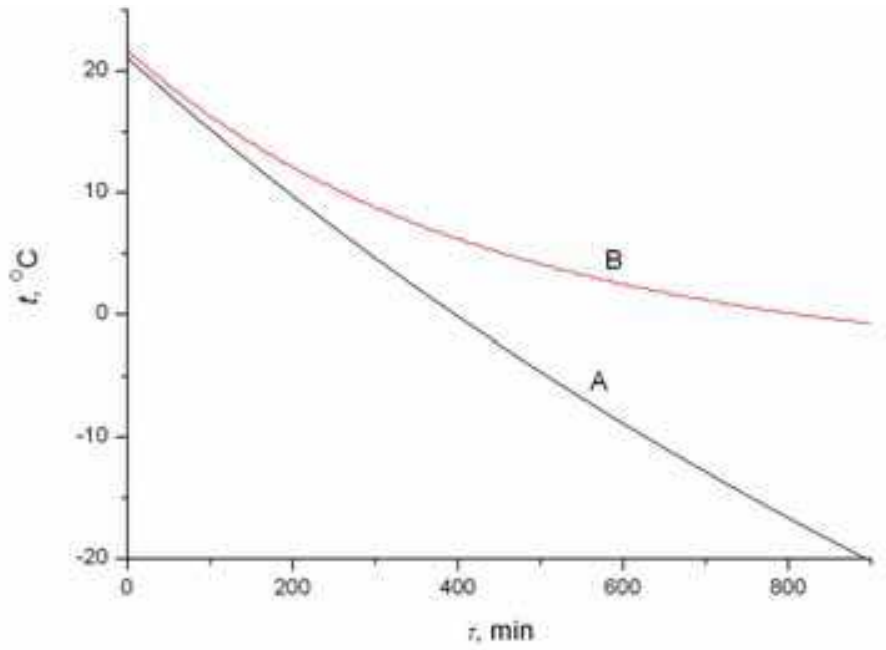

The systems supplied with radiators: one radiator (A), six radiators (B).

Fig. 5. Temperature drop of the thermal regulation object at a switch-off of electric power. 
The presented dependence shows that at an electric power switch-off the object temperature will drop from 21 to $-0.7^{\circ} \mathrm{C}$ when using six radiators and to $-20{ }^{\circ} \mathrm{C}$ when using a single radiator. At the operation of space equipment, a periodic considerable increase in power consumption by the equipment is also possible. In this case, it is necessary to use a radiator with an adjustable effective radiating area. Figure 6 shows the temperature variation of the regulation object at an increase in heat release from 10 to $20 \mathrm{~W}$ during 900 min while using a conventional system for ensuring the thermal regime and a system with an adjustable radiator.

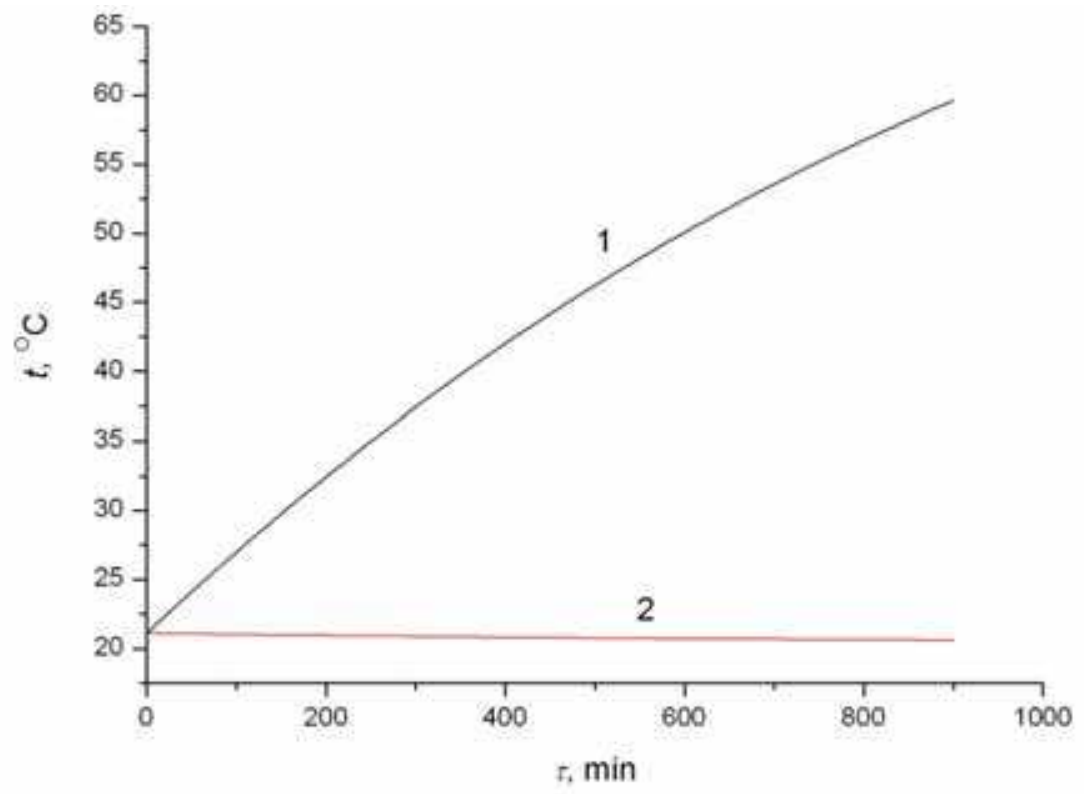

The systems supplied with radiators with constant (I) and adjustable (2) emissive areas.

Fig. 6. Temperature variation of the thermal regulation object with increasing power consumption.

It is seen from the presented dependence that the adjustable radiator is capable in this case of ensuring a nearly constant temperature of the equipment, whereas at the use of the conventional system the temperature increases from 21 to $60^{\circ} \mathrm{C}$.

Figure 7, which presents the temperature increase of a thermal regulation object cooled to $-100^{\circ} \mathrm{C}$ (the temperature typical of infrared and X-ray detectors) and mounted on the orbital spacecraft, demonstrates the efficiency of using thermal diodes in a low- temperature system at a turn by $90^{\circ}$ of the orbit plane with respect to the direction to the sun similarly to the turn shown in Fig. 4.

The given dependence shows that when the radiator-emitter orientation changes the temperature of the thermal regulation object cooled to $-100^{\circ} \mathrm{C}$ increases up to $-43^{\circ} \mathrm{C}$ during $1800 \mathrm{~mm}$ at the use of a standard system of thermal regulation and up to $-64{ }^{\circ} \mathrm{C}$ at the introduction of a thermal diode into the system. 


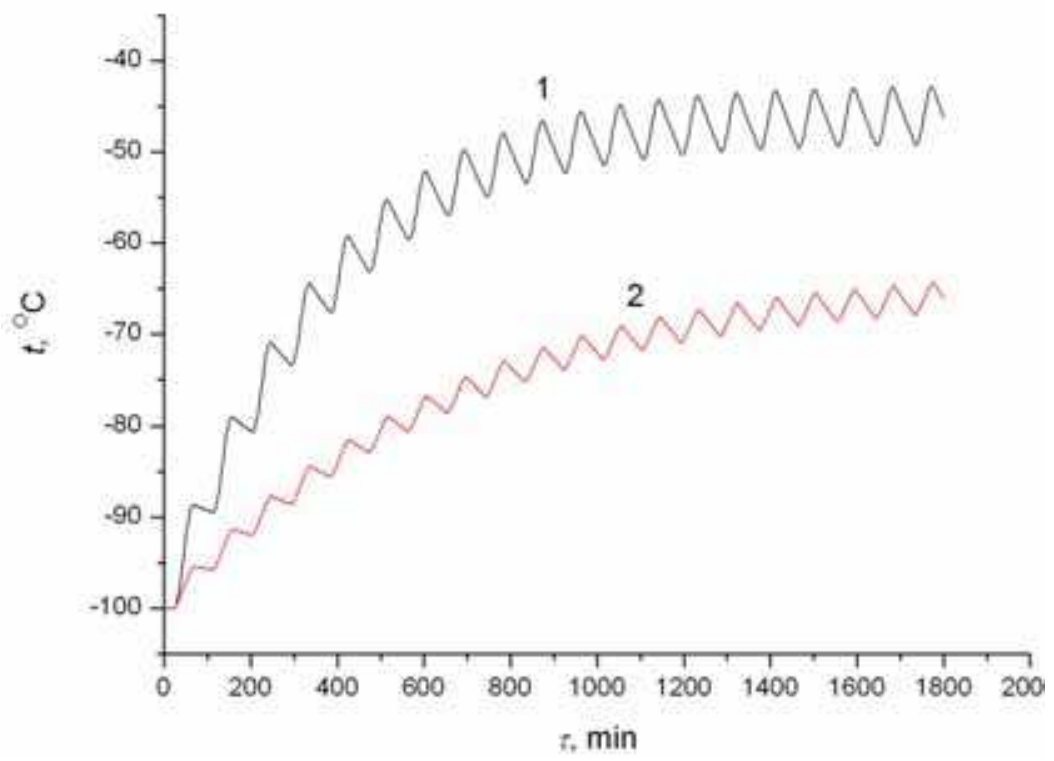

The systems supplied with radiators: with one radiator (1), one radiator and thermal diode (2).

Fig. 7. Temperature variation of the thermal regulation object cooled down to $-100{ }^{\circ} \mathrm{C}$ at a rotation of the spacecraft orbit.

\section{Use of space thermal factors for determination of the space vehicle orbit orietation}

In the previous sections the decisions have been shown, allowing to make Sc thermoregulation system the tolerant to anisotropy of a space thermal factors. But this anisotropy contains the information about a direction on external heat sources - the sun and a planet and hence, can be used for definition of Sc orientation.

As an example consider parameters of radiant flows in near-earth space. Specificity of the direct solar radiation is conditioned by significant remoteness of the Sun from Sc. In practical calculations the Sun can be considered as an infinitely distant radiation source. Therefore, its radiant flow over the near-earth orbit has characteristics that do not depend on the orbit parameters: constant small local divergence (32'); similar direction at a specific moment (solar radiation is parallel in volumes commensurable with Sc dimensions); constant irradiance $E s \sim 1400 \mathrm{~W} / \mathrm{m}^{2}$, with weak seasonal variations or zero intensity at Sc approaching the Earth shadow.

Self-radiation of the Earth, on the contrary, due to its proximity to Sc has characteristics depending on the actual height over the Earth: significant (up to $150^{\circ}$ ) angle of radiation divergence and irradiance up to $230 \mathrm{~W} / \mathrm{m}^{2}$. The Sun radiation reflected from the Earth also depends on the orbit height and, besides, on the time since intensity of such radiation depends on the variable in time positional relationship of the Sun, the Earth and the Sc.

Irradiance of Sc frame elements by direct solar radiation reflected from the Earth can vary in time at the orientation of the Sc to the Earth or its constant orientation to the Sun. Irradiation of various Sc frame elements by the Sun radiation reflected from the Earth is, vice versa, 
constant in time at Sc orientation to the Earth and variable at its orientation to the Sun. Spectrum distribution of direct and reflected from the Erath solar radiation lies mostly in the visible area. Self-radiation of the Earth is infrared.

The following shall be noted. If on the ground temperature of the external surface of any body is conditioned by a large number of random factors (wind velocity, air humidity, present nearby objects, soil temperature, etc.) in outer space the body orientation in relation to the Sun appears to be the principal external factor forming its surface field temperature.

To identify interdependence of orientation and thermal mode of Sc surface its thermal mathematical model can be used that is demonstrated by an example of the simplest located on the Earth orbit Sc being the cube with known conductive bounds between the facets (fig. 8).

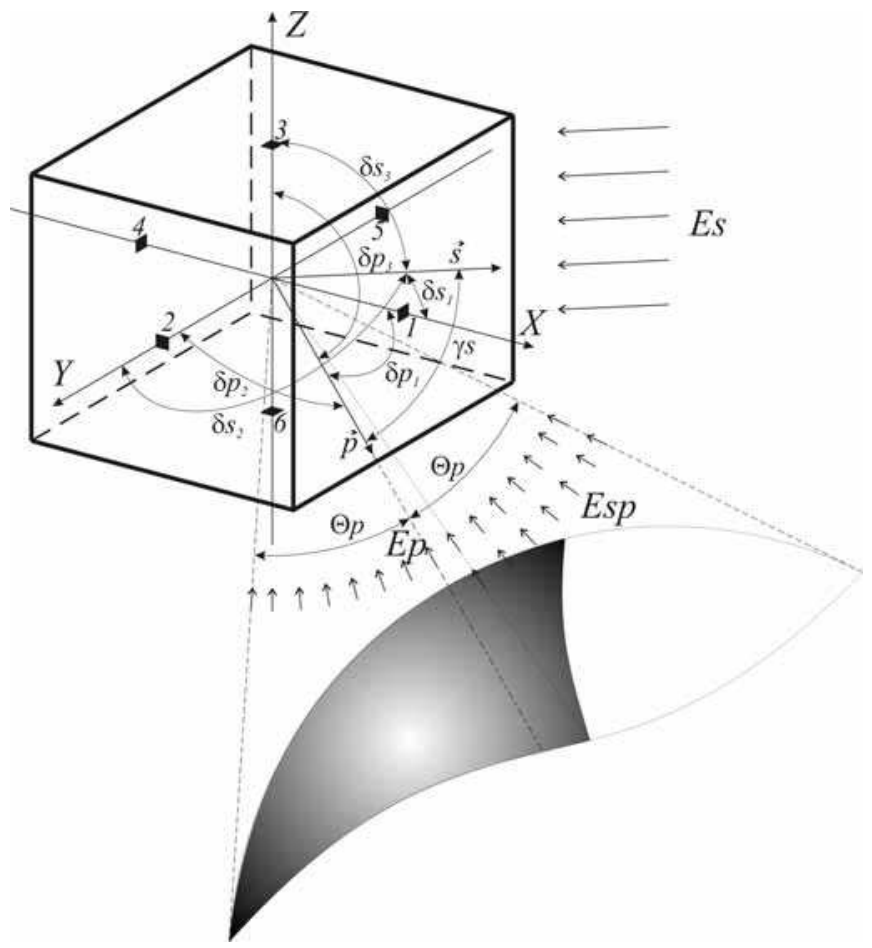

Fig. 8. Scheme of outer radiant flow effect on the near-earth cubical Sc.

Considering the cube facets as the thermal elements of Sc and assuming that Sc lacks internal heat generation and reradiation between the facets (more complex configuration and structure of the device would complicate the model having no impact on general results of the analysis), its thermal model will be described by the following six equations:

$$
\begin{aligned}
& C_{i} \cdot \frac{d T_{i}}{d \tau}=\left(\varepsilon_{i} \cdot E p_{i}+A s_{i} \cdot\left(E s_{i}+E s p_{i}\right)\right) \cdot s_{i}+ \\
& +\sum_{j=1(i \neq j)}^{6} \frac{T_{i}-T_{j}}{R_{i j}}-\varepsilon_{i} \cdot \sigma \cdot T_{i}^{4} \cdot s_{i} ; \\
& i=1 \ldots 6,
\end{aligned}
$$


where $C_{i}, T_{i}$ - values of heat capacity and temperature of six elements (cube facets), $R_{i j}$ heat resistance between $i$ - and $j$ - heat elements (cube facets), $A s_{i}, \varepsilon_{i}, S_{i}$ - values of the coefficients of solar radiation absorption and degree of blackness and area of six cube facets, $E s_{i}, E s p_{i}, E p_{i}$ - momentary values of irradiation by direct solar radiation, reflected from the Earth the solar radiation and self-radiation of the Earth of six cube facets, $\tau-$ time, $\sigma-$ Stefan-Bolzmann constant.

Directions to the principal heat sources - the Sun and the Earth - can be determined on the basis of eighteen values of the outer radiant flows $\left(E s_{i}, E s p_{i}, E p_{i}, i=1 \ldots 6\right)$. However, six equations of thermal model for their determination are not sufficient. Therefore, the thermal model shall be supplemented by no less than twelve equations. These equations can be obtained under the condition of all cube facets radiation by two sources (the Sun and the Earth) and constant mutual orientation of the facets in relation to each other.

Due to the specificity of direct solar radiation for the description of values $E s_{i}$ the simplest mathematical model can be applied. Choosing directions coinciding with normals to the first, second and third cube facets (see fig. 1 ) as axes $X, Y, Z$ of the coordinates system bound with Sc, we can draw up the following equations:

$$
\begin{aligned}
& E s_{i}=E s \cdot \cos \left(\delta s_{i}\right) \\
& i=1 \ldots 6,
\end{aligned}
$$

where $E s$ - normal irradiation by the solar radiation on the Earth orbit (the solar constant), $\delta s_{1}, \delta s_{2}, \delta s_{3}$ - angles between the positive directions of axes $X, Y, Z$ of the coordinates system bound with Sc and the direction to the Sun, $\delta s_{4}, \delta s_{5}, \delta s_{6}$ - angles between negative directions of axes $X, Y, Z$ of the coordinates system bound with Sc and the direction to the Sun.

Value Es has seasonal variations from 1396 to $1444 \mathrm{~W} / \mathrm{m}^{2}$, but at that, it is known with high degree of certainty for any day for a year.

Cube facets irradiation by self-radiation of the Earth $\left(E p_{1}, \ldots E p_{6}\right)$ is described in a more complex way:

$$
\begin{aligned}
& E p_{i}=\frac{1-A p}{4} \cdot E s \cdot \phi_{i} ; \\
& \phi_{i}=\cos \delta p_{i} \cdot \sin ^{2} \theta p \text { npu } 0 \leq \delta p_{i}<\frac{\pi}{2}-\theta p ; \\
& \phi_{i}=\frac{1}{\pi} \cdot\left(\cos \delta p_{i} \cdot \sin ^{2} \theta p \cdot\left(\frac{\pi}{2}-\arcsin \left(\operatorname{ctg} \theta p \cdot \operatorname{ctg} \delta p_{i}\right)\right)+\right. \\
& +\arcsin \frac{\sqrt{\sin ^{2} \theta p-\cos ^{2} \delta p_{i}}}{\sin \delta p_{i}}-\cos \theta p \cdot \sqrt{\sin ^{2} \theta p-\cos ^{2} \delta p_{i}} \\
& n p u \frac{\pi}{2}-\theta p \leq \delta p_{i}<\frac{\pi}{2}+\theta p ; \\
& \phi_{i}=0 \text { npu } \frac{\pi}{2}+\theta p \leq \delta p_{i} \leq \pi \\
& i=1 \ldots 6,
\end{aligned}
$$


where $A p$ - average albedo of the Earth, $\varphi_{i}$ - angular coefficient of irradiation of the $i$ facet of Sc by the planet, $\delta p_{1}, \delta p_{2}, \delta p_{3}$ - angles between positive directions $X, Y, Z$ of the coordinates system bound with $\mathrm{Sc}$ and the direction to the Earth center, $\delta p_{4}, \delta p_{5}, \delta p_{6}$ angles between negative directions of axes $X, Y, Z$ of the coordinates system bound with Sc and the direction to the center of the Earth, $\theta p$ - angle of view of the planet from the center of Sc.

The most complex model serves to describe cube facets irradiation by the solar radiation reflected from the Earth.

$$
\begin{aligned}
& E s p_{i}=A p \cdot E s \cdot \phi_{i}^{\prime} ; \\
& \phi_{i}^{\prime}=f_{2} \cdot \cos \delta p_{i} \cdot \cos \gamma s+f_{3} \cdot \sin \delta p_{i} \cdot \sin \gamma s \cdot \cos \beta s_{i} \\
& i=1 \ldots 6
\end{aligned}
$$

where $\varphi^{\prime}{ }_{i}$ - angular coefficient of irradiation of the part of the planet of $i$-facet of Sc, $f_{2}$ radiated by the Sun, $f_{3}$ - auxiliary functions, $\gamma_{s}$ - angle between directions to the Sun and to Sc from the planet center, $\beta s_{i}$ - two-facet angle with the vertex coinciding with the straight going through the planet center and Sc, in one plane of which lies the normal to $i-$ facet of Sc and in another one - direction to the Sun.

Functions $f_{2}, f_{3}$ are determined as follows.

If the plane of $i$-facet of Sc does not cross the planet:

$$
\begin{aligned}
& f_{2}(\theta p)=\frac{1}{4} \cdot\left(1+\sin ^{2} \theta p+2 \cdot \sin ^{3} \theta p+\frac{\cos ^{4} \theta p}{2 \cdot \sin \theta p} \cdot \ln \frac{1-\sin \theta p}{1+\sin \theta p}\right) \\
& f_{3}(\theta p)=\frac{\cos ^{2} \theta p \cdot\left(3+\sin ^{2} \theta p\right)}{16 \cdot \sin \theta p} \cdot \ln \frac{1+\sin \theta p}{1-\sin \theta p}- \\
& -\frac{(1-\sin \theta p) \cdot\left(3+3 \sin \theta p+2 \cdot \sin ^{2} \theta p\right)}{8}
\end{aligned}
$$

If the plane of $i$-facet of Sc crosses the planet:

$$
\begin{aligned}
& f_{2}\left(\theta p, \delta p_{i}\right)=\frac{f_{2}(\theta p)}{\sin ^{2} \theta p} \cdot \phi_{i} \\
& f_{3}\left(\theta p, \delta p_{i}\right)=f_{3}(\theta p) \text { npu } 0 \leq \delta p_{i} \leq \frac{\pi}{2}-\theta p \\
& f_{3}\left(\theta p, \delta p_{i}\right)=f_{3}(\theta p) \cdot \frac{\theta p+\frac{\pi}{2}-\delta p_{i}}{2 \cdot \theta p} n p u \frac{\pi}{2}-\theta p \leq \delta p_{i} \leq \frac{\pi}{2}+\theta p
\end{aligned}
$$

Missing equations can be obtained from the vector productions of unit vectors directed to the Sun $-\vec{s}\left(\cos \delta s_{1}, \cos \delta s_{2}, \cos \delta s_{3}\right)$, to the Earth $-\vec{p}\left(\cos \delta p_{1}, \cos \delta p_{2}, \cos \delta p_{3}\right)$, and orts $-\vec{e}_{x}(1,0,0), \vec{e}_{y}(0,1,0), \vec{e}_{z}(0,0,1)$ : 


$$
\begin{aligned}
& {\left[\vec{s} \cdot\left[\vec{e}_{x} \cdot \vec{e}_{y}\right]\right]=\vec{s} \cdot\left(\vec{e}_{x} \cdot \vec{e}_{y}\right)-\vec{e}_{x} \cdot\left(\vec{s} \cdot \vec{e}_{y}\right) ;} \\
& {\left[\vec{p} \cdot\left[\vec{e}_{x} \cdot \vec{e}_{y}\right]\right]=\vec{p} \cdot\left(\vec{e}_{x} \cdot \vec{e}_{y}\right)-\vec{e}_{x} \cdot\left(\vec{p} \cdot \vec{e}_{y}\right) ;} \\
& {\left[\vec{e}_{x} \cdot \vec{e}_{y}\right]=\vec{e}_{z} .}
\end{aligned}
$$

Thus, on the basis of the thermal mathematical model, the model that serves to calculate angles between the axes of the coordinates system bound with Sc and directions to the Sun $-\delta s_{i}$, and the planet $-\delta p_{i}$, i. e. to determine orientation of Sc.

Thermal model shows, that general measurement system of external Sc surface can use for determination of Sc orientation. This is possible at the condition of present plane multidirectional zones with the following properties:

- these zones shall have constant orientation in relation to the coordinates system bound with Sc;

- $\quad$ optical characteristics of these zones shall be known;

- there shall be no screening of these zones from the space by other structural elements;

- there shall be no less than six such zones, they shall be oriented so that each axis of the coordinates system bound with Sc form with the normal to the surface at least one angle less than 30 (theoretically less than 90) degrees in positive and negative directions;

- it is desirable that thermal sensitivity of these zones to the spatial external factors be much higher than to the internal heat factors of Sc;

- thermal capacity of these zones shall be minimum.

In some Sc such zones already exist. In the devices where such zones are difficult to part they can be created artificially as separate external elements set on the surface of the spacecraft [3].

In the Space Research Institute (SRI) RAS the model of such device has been developed and called a thermosensitive element (TSE).Orientation determination system based on thermosensitive elements is called thermodynamical system of orientation determination (TDSOD). The model of TSE has extremely reliable simple and inexpensive design. It consists of (fig. 9):

- $\quad$ heat receiving plate (1) from highly thermoconductive metal with special coating with calibrated optical characteristics;

- resistance thermometer (2), set in the center of the plate (1) so that thermal resistance between them be minimum;

- $\quad$ four-beam base (3), made of thin wall plate with low thermal conductivity (material stainless steel or titanium), at the same time being a thermal bypass between the thermosensitive plate and Sc frame;

- fiber-glass plastic base (4) with electric outputs connected with the resistance thermometer;

- thermoisolating screen (5), decreasing radiation flow from the structural elements to the heat receiving plate.

Thermosensitive elements of two types have been developed: solar and planetary (fig. 10), differing by optical characteristics of the heat receiving plate coating. Solar TSE was especially sensitive to the visible solar radiation for the account of the coating of "black nickel" type with As $=0,9$ and $\varepsilon=0,3$. Planetary TSE had heightened sensitivity to infrared radiation of the Earth for the account of the coating of AK-573 type with As $=0,2$ and $\varepsilon=0,8$. 


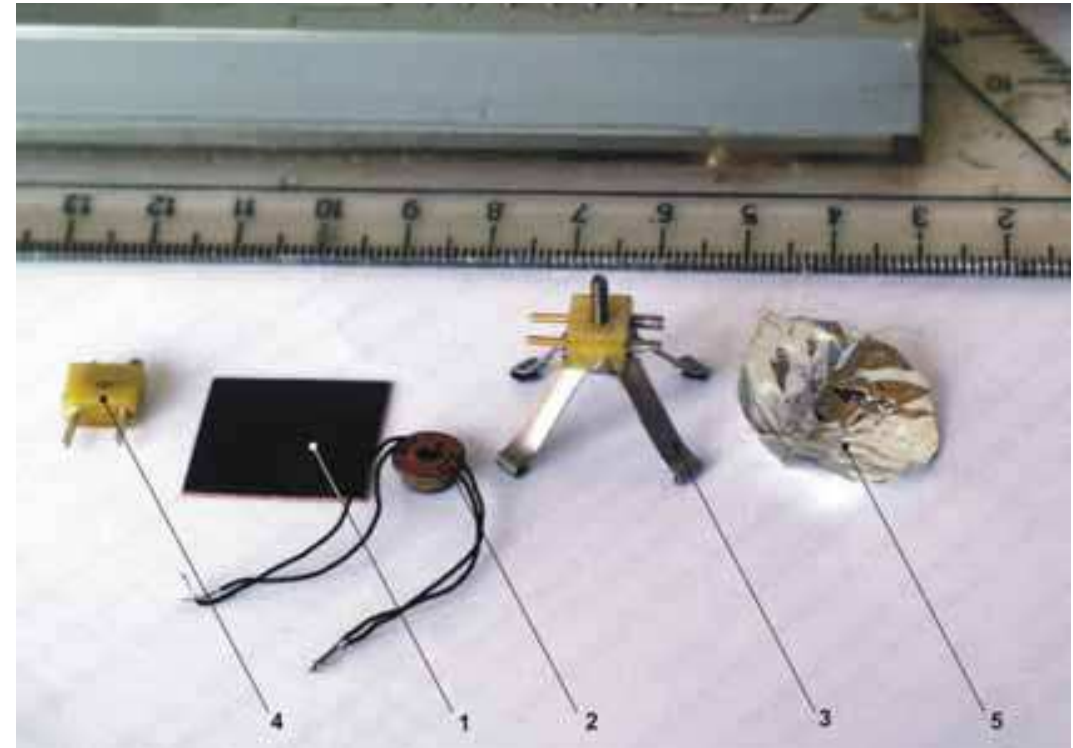

Fig. 9. TSE elements.

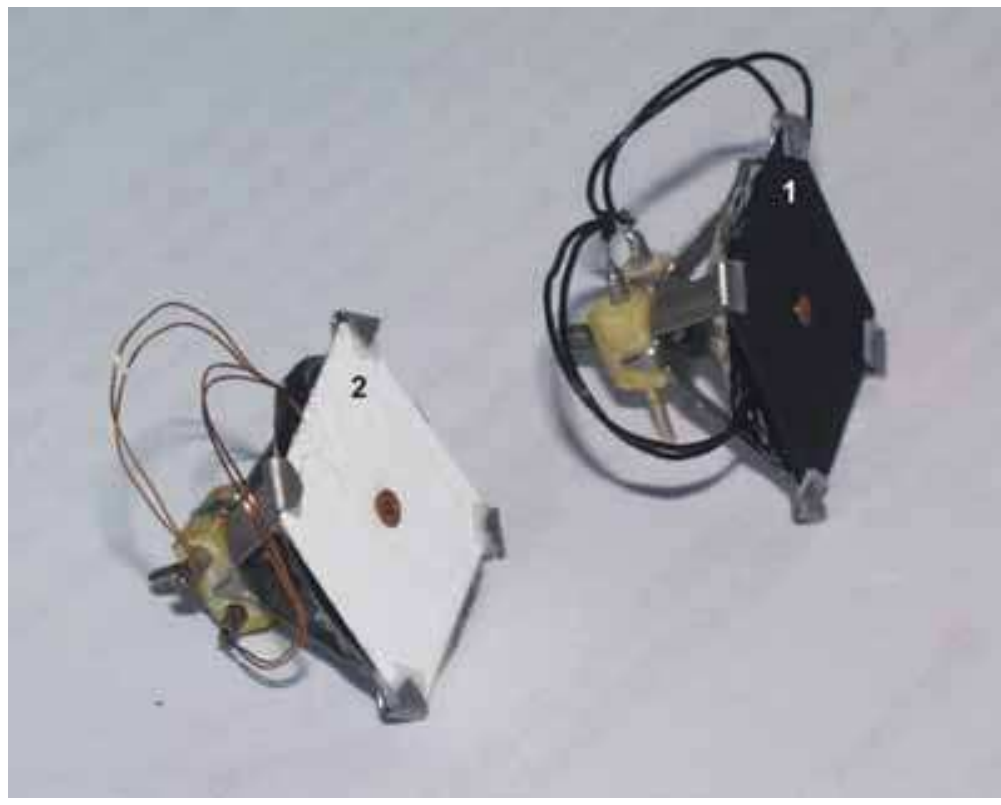

Fig. 10. General view of the solar (1) and planetary (2) TSE.

Thermodynamical system for orientation determination shall include not less than 12 such elements, one planetary and one solar perpendicular to each axis bound with Sc of the coordinates system in the positive and negative directions. 
To assess the possibility of TSE use as an element sensitive to the changing direction to the Sun eight models of TSE differing by the parameters of thermosensitive plates which characteristics given in the table experimental research has been carried out.

The TSE models were put into the vacuum chamber where main space thermal factors were imitated: "cold", "blackness", solar and planetary radiation. The models were placed on the rotary-support that served to turn TSE so that the angle between the normal to heat receiving plate and the direction to the Sun change in the range from -90 to $+90^{\circ}$. The device at the same time appeared to be an imitator of the Sc frame coated with screen-vacuum thermoisolation.

Initial angle between the normals to the heat receiving plates and the direction to the Sun was equal to $-90^{\circ}$. TSE turn from -90 to $+90^{\circ}$ was performed with different angular velocities imitating possible rotation of Sc.

Dependence of the temperature of the heat receiving plates (for basic TSE № 1 and № 2) from an angle between the normal to them and the direction to the Sun is presented in fig. 11: for rotation velocity 1,5 (a), $4(b), 10(c), 20(d), 30(e)$ ang.deg./min.

\begin{tabular}{|c|c|c|c|c|c|c|}
\hline \multirow{3}{*}{$\begin{array}{l}\text { TSE } \\
\text { № }\end{array}$} & \multicolumn{5}{|c|}{ Characteristics of the heat receiving plate } & \multirow{3}{*}{ TSE type } \\
\hline & \multirow[t]{2}{*}{ Size, $\mathrm{mm}$} & \multirow{2}{*}{$\begin{array}{c}\text { Thickness, } \\
\text { mm }\end{array}$} & \multirow[t]{2}{*}{ Material } & \multicolumn{2}{|c|}{$\begin{array}{l}\text { Optical characteristics of } \\
\text { the coating }\end{array}$} & \\
\hline & & & & $A s$ & $\varepsilon$ & \\
\hline 1 & $20 \times 20$ & 0,3 & Alloy Д16 & 0,9 & 0,3 & Solar \\
\hline 2 & $20 \times 20$ & 0,3 & Alloy Д16 & 0,2 & 0,8 & Planetary \\
\hline 3 & $20 \times 20$ & 0,5 & Alloy Д16 & 0,9 & 0,3 & Solar \\
\hline 4 & $20 \times 20$ & 0,5 & Alloy Д16 & 0,2 & 0,8 & Planetary \\
\hline 5 & $20 \times 20$ & 0,25 & Cuprum & 0,9 & 0,3 & Solar \\
\hline 6 & $20 \times 20$ & 0,25 & Cuprum & 0,9 & 0,3 & Solar \\
\hline 7 & $40 \times 40$ & 0,3 & Alloy Д16 & 0,9 & 0,3 & Solar \\
\hline 8 & $40 \times 40$ & 0,3 & Alloy Д16 & 0,2 & 0,8 & Planetary \\
\hline
\end{tabular}

Table 1. Characteristics of heat receiving plates of the studied TSE

Analysis of the presented experimental data shows that, first, the proposed TSE design appears to be rather sensitive for orientation determination in relation to the Sun, second, sensitivity of this method for orientation determination decreases at the increase of the velocity of Sc rotation in relation to the Sun due to the increase of thermal inertia of heat receiving plate.

To assess the possibility of orientation determination in relation to the Sun with the use of TSE it is expedient to introduce the parameter binding differential temperature and angular characteristics of TSE. Such parameter can be sensitivity $K$, showing temperature change of the heat receiving plate of the thermosensitive elements at their turn in relation to the Sun for one angular degree.

Dependence of TSE sensitivity $K$ on the angle $\alpha$ between the normal to the heat receiving plate and the direction to the Sun obtained on the basis of the experimental data is presented in fig. 12.

Thus, at the average TSE sensitivity equal to $1,2^{\circ} \mathrm{C} /$ ang.deg., determination accuracy of the direction to the Sun can be $5 \mathrm{arc}$ min. at the measurement accuracy of the temperature of $0,1^{\circ} \mathrm{C}$, that can be attained at the use of standard resistance thermometers and individual calibration of each TSE. 

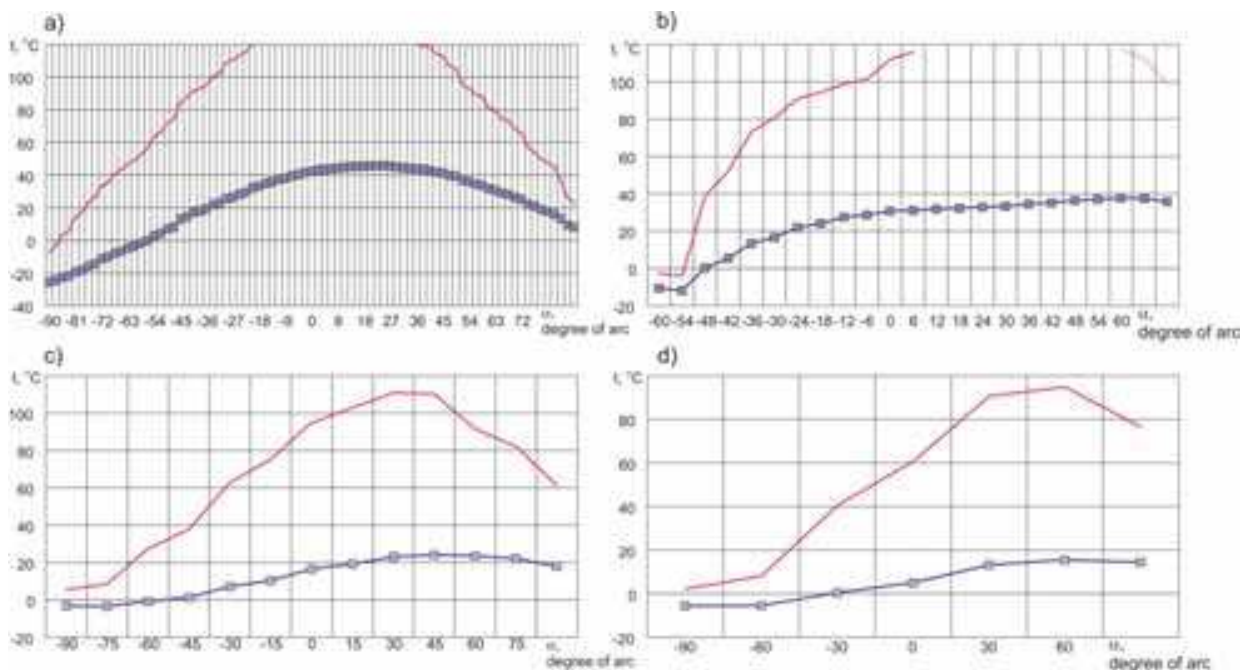

d)
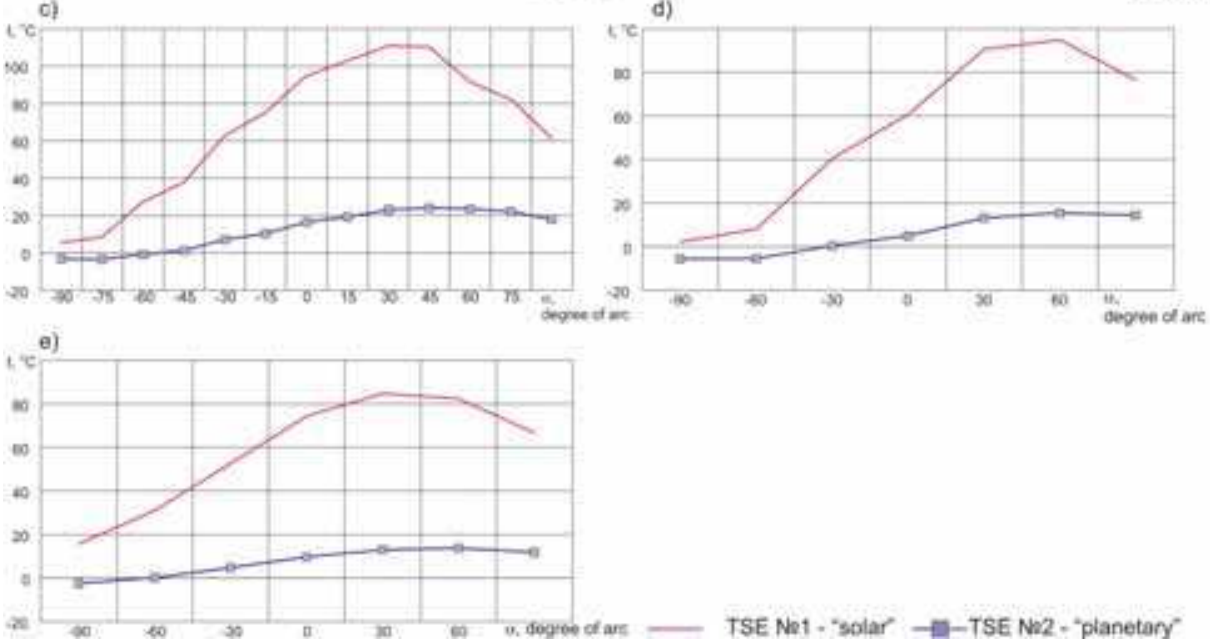

TSE Na1 - "solar"

TSE N22 - "planetary"

Fig. 11. Temperature dependence of the receiving plates of TSE № 1 and № 2 on the angle between the normal to them and the direction to the Sun.

$K,{ }^{\circ} \mathrm{C}$ ang $d$ eg.

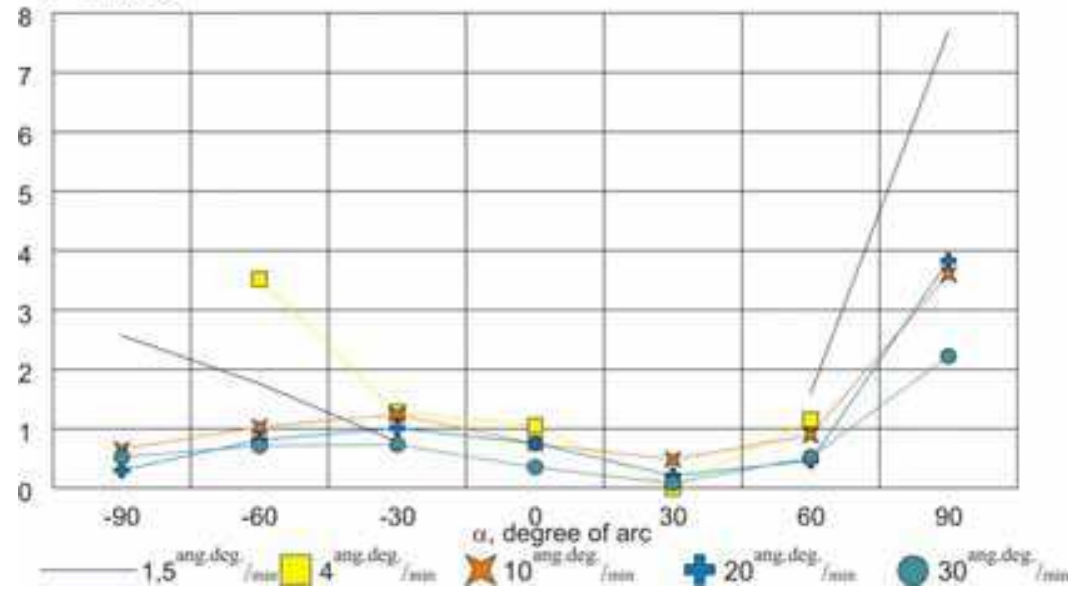

Fig. 12. Dependence of TSE sensitivity on the angle between the normal and heat receiving plate and the direction to the Sun. 
During the experiment we have also studied influence of the material, thickness and area of thermoconductive plate on its sensitivity. It has been found out that replacement of the aluminum alloy by cuprum at the production of the heat receiving plate practically has no impact on TSE sensitivity (fig. 13).

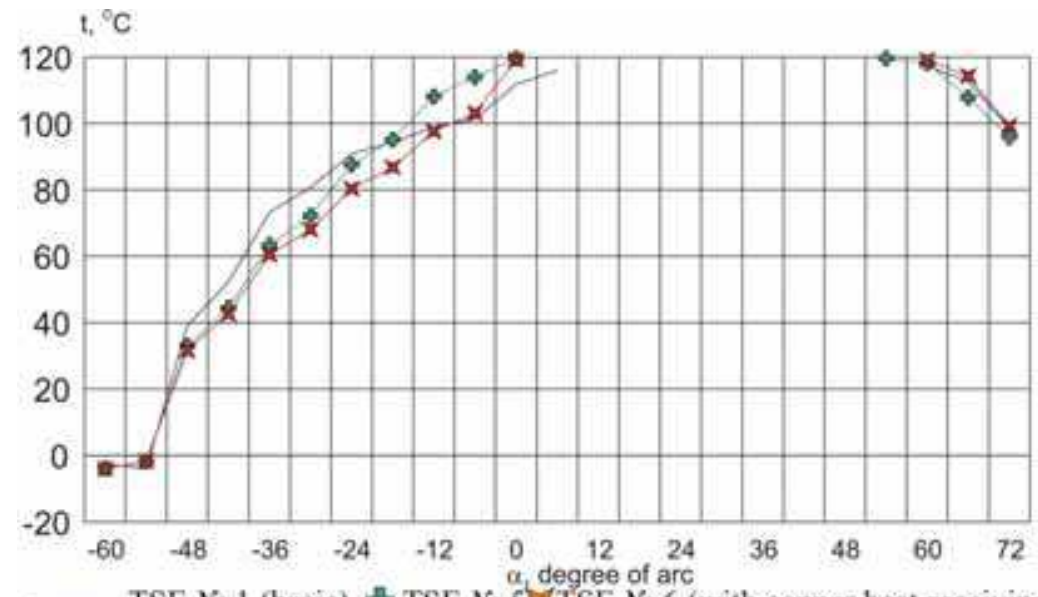

-TSE № 1 (basic) 둘 TSE № 5) TSE № 6 (with copper heat receiving plates)

Fig. 13. Temperature dependence of aluminum and copper heat receiving plates on the angle between the normal to them and the direction to the Sun.

Increase of the area of heat receiving plate enlarges TSE sensitivity at the angle between the normal to the plate and the direction to the Sun of less than $45^{\circ}$ (fig. 14). Increase of the thickness of the heat receiving plate significantly reduces its reaction on the changing direction of the solar radiation (fig. 15).

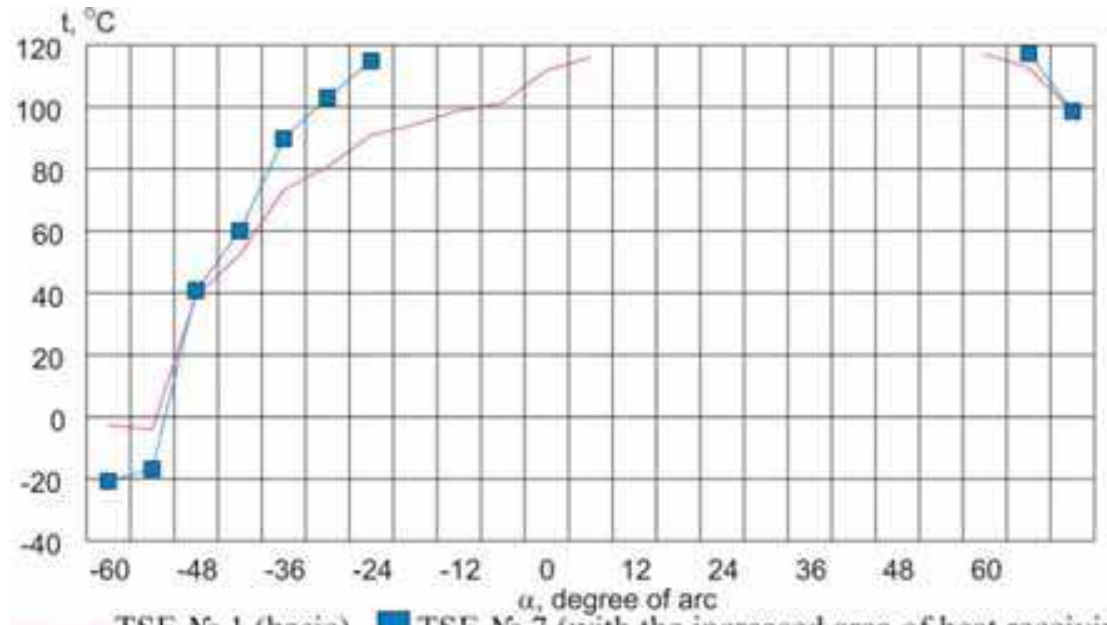

TSE No 1 (basic)

Fig. 14. Dependence of the temperature of heat receiving plates with the dimensions $20 \times 20$ and $40 \times 40 \mathrm{~mm}$ on the angle between the normal to them and the direction to the Sun. 


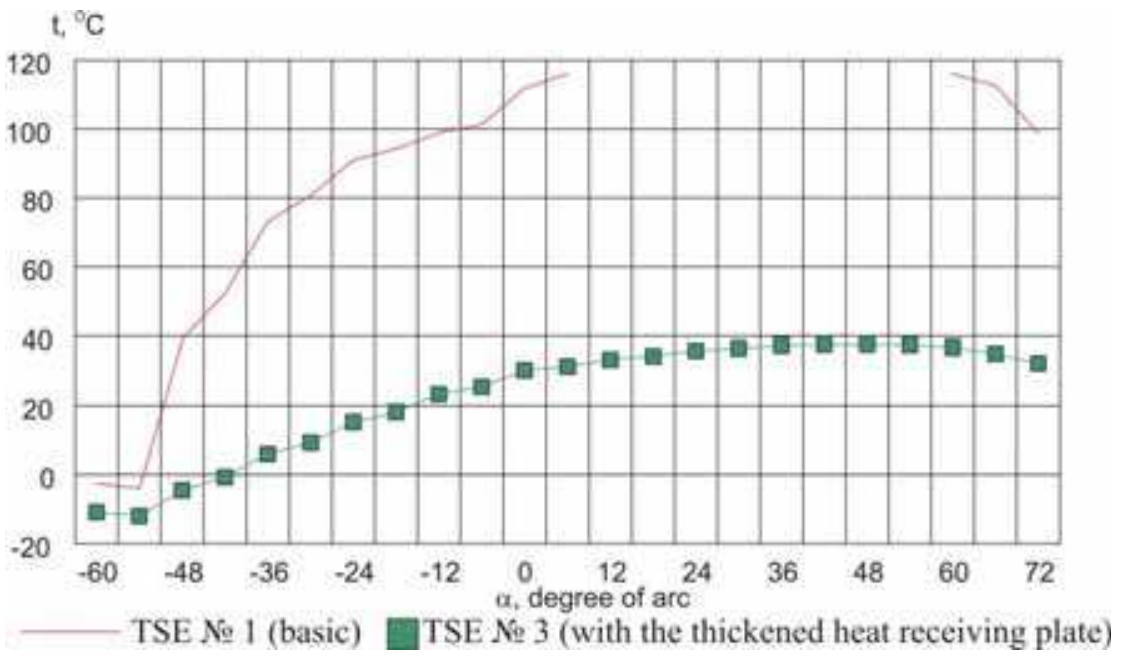

Fig. 15. Temperature dependence of heat receiving plates with the thickness of 0,3 and 0,5 $\mathrm{mm}$ on the angle between the normal to them and the direction to the Sun.

\section{Conclusion}

Usually the spacecraft have heat insulation from a space as much as possible. Heat, generated in the spacecraft, irradiate in the space through radiators - small open zone of an external surface. For additional heating of the spacecraft the heaters are provided.

However in Solar system is as heating sources (the sun and planets), so cooling sources (space). Use of several differently oriented radiators allows creating self-regulated thermal system of spacecraft on the balance between the absorbed irradiance from external heat source and the radiated thermal flux. Such system doesn't need energy and works at any spacecraft orientation. Besides, this system allows defining a direction on the Sun and the Earth and therefore can be used for definition of spacecraft orientation.

\section{References}

[1] Semena N.P., Konovalov A.A. Methods for creating the self-regulating mechanisms of passive systems for ensuring thermal regime of devices for space application, Thermophysics and Aeromechanics, 2007, Vol. 14, No. 1, p.81-91.

[2] Semena N.P. Determination of spacecraft orientation by the temperature field analysis of its outer surface, Thermophysics and Aeromechanics, 2009, Vol. 16, No 1, p.135-143.

[3] Semena N.P., Kostenko V.I., Logvinenko S.P. Determination of the Spacecraft Angular Position by Temperature Measurements of Thermosensitive Plates, RF Patent № 2126137, 10 February 1999. 


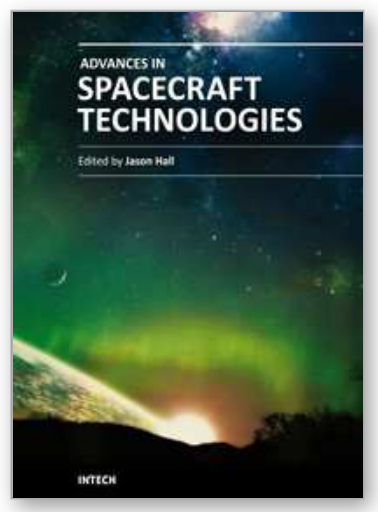

\author{
Advances in Spacecraft Technologies \\ Edited by Dr Jason Hall
}

ISBN 978-953-307-551-8

Hard cover, 596 pages

Publisher InTech

Published online 14, February, 2011

Published in print edition February, 2011

The development and launch of the first artificial satellite Sputnik more than five decades ago propelled both the scientific and engineering communities to new heights as they worked together to develop novel solutions to the challenges of spacecraft system design. This symbiotic relationship has brought significant technological advances that have enabled the design of systems that can withstand the rigors of space while providing valuable space-based services. With its 26 chapters divided into three sections, this book brings together critical contributions from renowned international researchers to provide an outstanding survey of recent advances in spacecraft technologies. The first section includes nine chapters that focus on innovative hardware technologies while the next section is comprised of seven chapters that center on cutting-edge state estimation techniques. The final section contains eleven chapters that present a series of novel control methods for spacecraft orbit and attitude control.

\title{
How to reference
}

In order to correctly reference this scholarly work, feel free to copy and paste the following:

Nikolay Semena (2011). Use of Space Thermal Factors by Spacecraft, Advances in Spacecraft Technologies, Dr Jason Hall (Ed.), ISBN: 978-953-307-551-8, InTech, Available from:

http://www.intechopen.com/books/advances-in-spacecraft-technologies/use-of-space-thermal-factors-byspacecraft

\section{INTECH}

open science | open minds

\section{InTech Europe}

University Campus STeP Ri

Slavka Krautzeka 83/A

51000 Rijeka, Croatia

Phone: +385 (51) 770447

Fax: +385 (51) 686166

www.intechopen.com

\section{InTech China}

Unit 405, Office Block, Hotel Equatorial Shanghai

No.65, Yan An Road (West), Shanghai, 200040, China

中国上海市延安西路65号上海国际贵都大饭店办公楼 405 单元

Phone: +86-21-62489820

Fax: $+86-21-62489821$ 
(C) 2011 The Author(s). Licensee IntechOpen. This chapter is distributed under the terms of the Creative Commons Attribution-NonCommercialShareAlike-3.0 License, which permits use, distribution and reproduction for non-commercial purposes, provided the original is properly cited and derivative works building on this content are distributed under the same license. 